\title{
Stationary solutions and self-trapping in discrete quadratic nonlinear systems
}

\author{
Bang, Ole; Christiansen, Peter Leth; Clausen, Carl A. Balslev
}

Published in:

Physical Review E. Statistical, Nonlinear, and Soft Matter Physics

Link to article, DOI:

10.1103/PhysRevE.56.7257

Publication date:

1998

Document Version

Publisher's PDF, also known as Version of record

Link back to DTU Orbit

Citation (APA):

Bang, O., Christiansen, P. L., \& Clausen, C. A. B. (1998). Stationary solutions and self-trapping in discrete quadratic nonlinear systems. Physical Review E. Statistical, Nonlinear, and Soft Matter Physics, 56(6), 72577266. https://doi.org/10.1103/PhysRevE.56.7257

\section{General rights}

Copyright and moral rights for the publications made accessible in the public portal are retained by the authors and/or other copyright owners and it is a condition of accessing publications that users recognise and abide by the legal requirements associated with these rights.

- Users may download and print one copy of any publication from the public portal for the purpose of private study or research.

- You may not further distribute the material or use it for any profit-making activity or commercial gain

- You may freely distribute the URL identifying the publication in the public portal 


\title{
Stationary solutions and self-trapping in discrete quadratic nonlinear systems
}

\author{
Ole Bang \\ Australian Photonics Cooperative Research Center, Research School of Physical Sciences and Engineering, \\ Optical Sciences Centre, Australian National University, Canberra, Australian Capital Territory 0200, Australia \\ Peter Leth Christiansen and Carl Balslev Clausen \\ Department of Mathematical Modelling, Technical University of Denmark, DK 2800 Lyngby, Denmark
}

(Received 21 July 1997)

\begin{abstract}
We consider the simplest equations describing coupled quadratic nonlinear $\left(\chi^{(2)}\right)$ systems, which each consists of a fundamental mode resonantly interacting with its second harmonic. Such discrete equations apply, e.g., to optics, where they can describe arrays of $\chi^{(2)}$ waveguides, and to solid state physics, where they can describe nonlinear interface waves under the conditions of Fermi resonance of the adjacent crystals. Focusing on the monomer and dimer we discuss their Hamiltonian structure and find all stationary solutions and their stability properties. In one limit the nonintegrable dimer reduce to the discrete nonlinear Schrödinger (DNLS) equation with two degrees of freedom, which is integrable. We show how the stationary solutions to the two systems correspond to each other and how the self-trapped DNLS solutions gradually develop chaotic dynamics in the $\chi^{(2)}$ system, when going away from the near integrable limit.

[S1063-651X(97)05512-8]
\end{abstract}

PACS number(s): 63.20.Ry, 63.20.Pw, 42.65.Wi, 42.65.Sf

\section{INTRODUCTION}

Coupled nonlinear ordinary differential equations (ODE's) are used in the description of many physical problems. Such sets of equations can describe the dynamics in inherently discrete systems, such as coupled anharmonic oscillators or molecules in condensed matter physics, or waveguide arrays in nonlinear optics. They can also be viewed as a discretization of a corresponding continuous "field" equation.

One of the most studied systems of coupled nonlinear ODE's is the discrete self-trapping (DST) equation [1],

$$
i \partial_{\xi} W_{n}+\sum_{m \neq n}\left(J_{n m} W_{m}\right)+\left|W_{n}\right|^{2} W_{n}=0,
$$

where $\xi$ is the evolution coordinate, and $n=\left[1, n_{0}\right]$, with $n_{0}$ being the total number of sites. Classically the DST equation describes the dynamics of $n_{0}$ linearly coupled anharmonic oscillators with complex mode amplitudes $W_{n}(\xi)$. The strength of the coupling between sites $n$ and $m$ is given by $J_{n m}$. The DST equation describes a variety of effects in condensed matter physics, such as polarons [2], excitons in molecular chains [3], stretching vibrations of the hydrogen bonds in small polyatomic molecules such as water, ammonia, methane, and benzene [4], self-trapping of vibrational energy in hydrogen bonded polypeptide crystals such as acetanilide [5] and $\mathrm{N}$-methylacetamide [6], and globular protein [7].

The DST equation is a generalization of the discrete nonlinear Schrödinger (DNLS) equation

$$
i \partial_{\xi} W_{n}+\rho\left(W_{n+1}+W_{n-1}\right)+\left|W_{n}\right|^{2} W_{n}=0,
$$

the difference being that the DST equation takes into account arbitrary linear coupling between all the sites through the
$J_{n m}$ 's, whereas only nearest neighbor coupling $(\rho)$ is taken into account in the DNLS equation. Besides condensed matter physics the DNLS equation is also widely used in nonlinear optics, where it can describe wave propagation in arrays of cubic nonlinear waveguides [8-10]. In that case $W_{n}(\xi)$ represents the slowly varying envelope of a weakly modulated carrier wave.

The DNLS equation is generic and represents the simplest possible model for coupled cubic nonlinear oscillators with only a single frequency. Here we will consider the simplest model for coupled quadratic nonlinear oscillators, each with two frequencies, a fundamental $\left(W_{n}\right)$ and a second harmonic $\left(V_{n}\right)$, close to resonance

$$
\begin{gathered}
i \partial_{\xi} W_{n}+\eta_{w}\left(W_{n+1}+W_{n-1}\right)+W_{n}^{*} V_{n}=0, \\
i \partial_{\xi} V_{n}+\eta_{v}\left(V_{n+1}+V_{n-1}\right)-\alpha V_{n}+W_{n}^{2} / 2=0,
\end{gathered}
$$

where $\eta_{w}\left(\eta_{v}\right)$ determines the strength of the coupling between fields $W_{n}\left(V_{n}\right)$ at neighboring sites. The phase mismatch $\alpha$ determines how far the two fields are from resonance. The system (1.3-1.4) is used in solid state physics to describe nonlinear interface waves between two media close to Fermi resonance $[11,12]$, and in optics to describe arrays of quadratic nonlinear waveguides [13]. For only one site, $n_{0}=1$, Eqs. (1.3-1.4) reduce to simple second harmonic generation, which is one of the earliest and most well-studied effects in nonlinear optics [14].

Studying the system (1.3-1.4) is important, not only in terms of fundamental physics, but also from a technological point of view. In optics the DNLS equation is the simplest model for beam propagation in arrays of cubic (or $\chi^{(3)}$ ) nonlinear waveguides with a centrosymmetric crystal structure [9]. One of the most interesting effects of the cubic nonlinearity is that the index of refraction becomes dependent on the intensity. This is known as the Kerr effect and leads to 
self-action processes, such as self-phase modulation and selftrapping (or self-focusing in continuum media), which can be used in all optical signal processing [15]. However, in conventional materials the third order Kerr nonlinearity is weak and relatively slow (see [15] for a review). By using the generally stronger and faster second order quadratic (or $\chi^{(2)}$ ) nonlinearity in noncentrosymmetric materials instead, these obstacles can be overcome.

It is now well known that quadratic materials have effective cubic properties, such as an intensity dependent refractive index and self-phase modulation. The physical mechanism behind these effects is known as cascading, because the fundamental and second harmonic wave components interact with themselves through repeated up and down conversion (see [16] for a comprehensive review on cascading and its application to all-optical signal processing). However, it is only in certain limits that the cascading nonlinearity can be treated as an effective cubic nonlinearity. Generally the $\chi^{(2)}$ materials display a much richer variety of phenomena than can be found in $\chi^{(3)}$ materials [16]. As we will briefly show, the simplest model for beam propagation in arrays of quadratic nonlinear waveguides is given by Eqs. (1.3-1.4). Thus it is important to understand the properties of the system (1.3-1.4), in order to be able to fully utilize the potential of quadratic nonlinearity.

Furthermore, the system (1.3-1.4) is important from the context of being the simplest discretization of the continuum equations. In the continuum limit Eq. (1.1) becomes the onedimensional (1D) NLS equation, which is integrable and has stable soliton solutions [17]. In higher dimensions the solitary wave solutions to the NLS equation are unstable and can collapse in finite time (see [18] for a general review). In contrast the continuum counterpart to Eqs. (1.3-1.4) has stable solitary wave solutions in all dimensions of physical interest [19-21], and regardless of the initial wave function, a catastrophic collapse can never occur [19]. Due to the nature of the cascaded nonlinearity, the solitary waves in quadratic materials differ from the NLS soliton, in that they have two components, the fundamental wave and its second harmonic. Experimentally, two-component solitary waves have been observed in both 2D [22] and 1D [23].

Since quadratic materials have effective cubic properties there is obviously a connection between the two kinds of nonlinearities. Indeed it is possible to derive an NLS equation for the fundamental wave in quadratic materials, using perturbation techniques [24]. The connection may also be seen directly from the discrete Eqs. (1.3-1.4) by assuming that the phase mismatch is large, $|\alpha| \gg 1$, while the derivatives of $V_{n}$ remain finite. In that case the second harmonic field is weak and slaved to the fundamental, $V_{n} \approx W_{n}^{2} /(2 \alpha)$, with $W_{n}$ being determined by the DNLS equation.

The properties of the continuum equations corresponding to Eqs. (1.3-1.4) are well known; it has a continuous oneparameter family of bright solitary wave solutions [25], which is stable, except for only a narrow region in parameter space [21]. A lot less is known about the discrete Eqs. (1.31.4). The main work has been done in the context of Fermi resonance interface waves $[11,12]$, with only a single work published in nonlinear optics [13]. In general the focus has been on systems with many sites $\left(n_{0} \gg 1\right)$, in which localized self-trapped states of different topology have been found to exist, that at low amplitude can move through the lattice and either fuse, annihilate, or pass through each other when colliding [13]. In contrast to the DNLS equation, whose continuum limit is integrable, these collision properties are also found in the nonintegrable continuum limit of Eqs. (1.3-1.4) [26].

Even for the more conventional cubic nonlinear waveguides, the largest array fabricated to date consists of only 11 waveguides [27]. In the context of nonlinear optics it therefore seems most appropriate to study systems with only a few sites. Here we consider the coupler, or so-called dimer, with $n_{0}=2$, which has not yet been analyzed in detail. The $\chi^{(2)}$ dimer is nonintegrable, but in the limit of a large phase mismatch, $|\alpha| \gg 1$, it reduces to the DNLS dimer, which is integrable [1]. Thus it is naturally to compare the properties of the two systems, which we do throughout the paper. In most cases no analytical forms for solutions of Eqs. (1.31.4 ), or the DST or DNLS equations, are known. However, there are a physically important subclass of solutions that can be easily classified and often expressed in a simple form. These are the stationary solutions, which we therefore focus on in the comparison. We consider the system from the point of view of optics and thus we will talk about $\mathrm{cw}$ beams, envelope functions, and waveguides, etc.

In Sec. II we heuristically derive the model and give its main features, such as conserved quantities, Hamiltonian structure, and symmetries. In Secs. III A and III B we briefly consider the stationary solutions and fixed points of the monomer, $n_{0}=1$. These are of course known from the theory of second harmonic generation [14], but we will need them, since in certain cases the dimer can be reduced to the monomer. Furthermore, in Sec. III C, we discuss the Hamiltonian structure of the monomer, and show how it can be written as a compact su(2) algebra, in a similar way as for the DST equation [28]. The dimer is treated in Sec. IV. In particular we find all the stationary solutions analytically and analyze their stability properties in Sec. IV A. In Sec. IV B we then consider their self-trapping properties, and how they are connected to the stationary solutions of the DNLS dimer when $|\alpha| \gg 1$. Since the DNLS dimer is integrable, we can talk about a near-integrable limit of the $\chi^{(2)}$ dimer. An interesting question is then how the stationary DNLS solutions, when used as initial conditions in the $\chi^{(2)}$ system, will develop dynamics when going away from this limit. This is also considered in Sec. IV B, where we show how a gradual transition to chaos occurs. Finally, we discuss the Hamiltonian structure in Sec. IV C, and finish with a conclusion in Sec. V.

Before proceeding, we would like to draw attention to a recent paper by Dubovskii and Orlov [12], in which they analytically find stationary solutions of Eqs. (1.3-1.4) for $n_{0}=2,4$, and 6 . However, they consider only real solutions, and do not analyze stability. This means that for $n_{0}=2$, they only find two of the all in all seven different classes of solutions that we find here.

\section{THE MODEL}

Neglecting spatial walk-off, the dimensionless equations for $\mathrm{cw}$ beams in a lossless waveguide are the same as for 


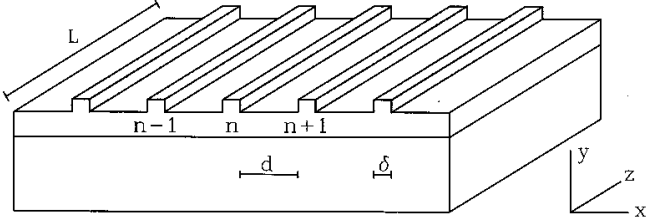

FIG. 1. Schematic diagram of part of an array of waveguides of length $L$. The width of each waveguide is $\delta$ and the spacing between waveguides is $d$.

second harmonic generation [14],

$$
i \partial_{z} \mathcal{W}+\chi \mathcal{W} * \mathcal{V} e^{-i \beta z}=0, \quad i \sigma \partial_{z} \mathcal{V}+2 \chi \mathcal{W}^{2} e^{i \beta z}=0
$$

where the complex functions $\mathcal{W}(z)$ and $\mathcal{V}(z)$ are the lowamplitude, slowly varying envelopes of the fundamental wave and its second harmonic, respectively. The parameter $\beta$ is proportional to the phase mismatch $\Delta k=2 k_{1}-k_{2}, \sigma=$ $k_{2} / k_{1} \approx 2$ is the ratio between the wave numbers, and $\chi$ is the normalized appropriate component of the $\chi^{(2)}$ susceptibility. The mode structure in the transverse $(x, y)$ plane is determined separately (see, e.g., [29]).

Let us now consider an array of $n_{0}$ identical regularly spaced quadratic nonlinear wave guides, as depicted in Fig. 1. Assume the distance between waveguides, $d$, to be large enough to allow the field in each waveguide to be treated almost as though in isolation. By almost we mean that $d$ should still be sufficiently small to allow the evanescent field tails in neighboring waveguides to overlap just enough to create a small linear coupling (power leakage). Under these assumptions, to lowest order, the stationary envelope of the electric field in the $n$th waveguide is governed by the equations

$$
\begin{gathered}
i \partial_{z} \mathcal{W}_{n}+\rho\left(\mathcal{W}_{n+1}+\mathcal{W}_{n-1}\right)+\chi \mathcal{W}_{n}^{*} \mathcal{V}_{n} e^{-i \beta z}=0 \\
i \sigma \partial_{z} \mathcal{V}_{n}+\sigma \kappa\left(\mathcal{V}_{n+1}+\mathcal{V}_{n-1}\right)+2 \chi \mathcal{W}_{n}^{2} e^{i \beta z}=0
\end{gathered}
$$

where $n=\left[1, n_{0}\right]$, and $\rho$ and $\kappa$ determine the coupling between adjacent waveguides for the fundamental and second harmonic, respectively.

In order to write the equations in a convenient dimensionless Hamiltonian form, we apply the transformation

$$
\mathcal{W}_{n}(z)=\left(\frac{\rho \sqrt{\sigma}}{2 \chi}\right) W_{n}(\zeta), \quad \mathcal{V}_{n}(z)=\left(\frac{\rho}{\chi}\right) V_{n}(\zeta) e^{i \beta z},
$$

where $\zeta=\rho z$. This reduces Eqs. $(2.1-2.2)$ to

$$
\begin{gathered}
i \dot{W}_{n}+\left(W_{n+1}+W_{n-1}\right)+W_{n}^{*} V_{n}=0, \\
i \dot{V}_{n}+\eta\left(V_{n+1}+V_{n-1}\right)-\alpha V_{n}+W_{n}^{2} / 2=0,
\end{gathered}
$$

where the dot denotes differentiation with respect to $\zeta$. We have assumed here that $\rho \neq 0$, in order to reduce the number of parameters in the model to two: the relative coupling strength $\eta=\kappa / \rho$, and the normalized phase mismatch $\alpha=$ $\beta / \rho$. The system (2.3-2.4) conserves the norm $N$ (dimensionless power) and the Hamiltonian $H$ :

$$
\begin{gathered}
N=\sum_{n}\left[\left|W_{n}\right|^{2}+2\left|V_{n}\right|^{2}\right], \\
H=\sum_{n}\left[\alpha\left|V_{n}\right|^{2}-W_{n}^{*}\left(W_{n+1}+W_{n-1}\right)-\eta V_{n}^{*}\left(V_{n+1}+V_{n-1}\right)\right. \\
\left.-\operatorname{Re}\left\{W_{n}^{2} V_{n}^{*}\right\}\right] .
\end{gathered}
$$

It is Hamiltonian with the conjugate variables $\left(W_{n}, i W_{n}^{*}\right)$ and $\left(V_{n}, i V_{n}^{*}\right)$ and can be written in the standard form $i \dot{W}_{n}=$ $\partial H / \partial W_{n}^{*}, i \dot{V}_{n}=\partial H / \partial V_{n}^{*}$. Furthermore, it is invariant under the transformation

$$
W_{n}(\zeta) \rightarrow W_{n}(\zeta) e^{i \Omega}, \quad V_{n}(\zeta) \rightarrow V_{n}(\zeta) e^{i 2 \Omega},
$$

where $\Omega$ is an arbitrary constant phase. This will be useful when considering stationary solutions, since it allows us to assume that one of the fields is real and positive.

\section{ONE WAVEGUIDE - THE MONOMER}

In this section we consider a single waveguide, corresponding to simply second harmonic generation $[14,30]$. Then Eqs. (2.3-2.4) reduce to the system of two equations

$$
i \dot{W}_{1}+W_{1}^{*} V_{1}=0, \quad i \dot{V}_{1}-\alpha V_{1}+W_{1}^{2} / 2=0,
$$

which is integrable and can be compared to the integrable DST dimer (same number of degrees of freedom) [1]. We first map the regions of existence and stability of all stationary solutions to Eqs. (3.1) (Sec. III A). Then we reduce the equations to the simplest possible Hamiltonian system of 2 degrees of freedom, and characterize the fixed points and dynamical behavior (Sec. III B). Finally we discuss the Hamiltonian structure of the equations in Sec. III C and show how they can be written in terms of Feynman variables that satisfy a compact su(2) algebra, in a similar way as for the DST equation [28].

\section{A. Stationary solutions}

The stationary solutions are defined as

$$
W_{1}(\zeta)=w_{1} e^{i \lambda \zeta}, \quad V_{1}(\zeta)=v_{1} e^{i 2 \lambda \zeta},
$$

where the eigenvalue $\lambda$ is real. The amplitude $w_{1}$ can be assumed to be real and non-negative, because of the scaling property given by Eq. (2.7). Inserting Eq. (3.2) into Eqs. (3.1) we find two nonzero solutions. The solution

$$
\text { (I) } \quad w_{1}=0, \quad\left|v_{1}\right|^{2}=N / 2
$$

exists at $\lambda=-\alpha / 2$, whereas the solution

$$
\text { (II) } \quad w_{1}^{2}=2 \lambda(\alpha+2 \lambda), \quad v_{1}=\lambda
$$

exists for $\lambda<\min \{0,-\alpha / 2\}$ and $\lambda>\max \{0,-\alpha / 2\}$. The zero solution $w_{1}=v_{1}=0$ exists for all $\lambda$, but here and in the following we will not consider this solution.

We have depicted the solutions (I) and (II) in Figs. 2(a) and 2(b). From Fig. 2(a) we see that except at exact phase matching, $\alpha=0$, there is a gap in the eigenvalue spectrum, in 

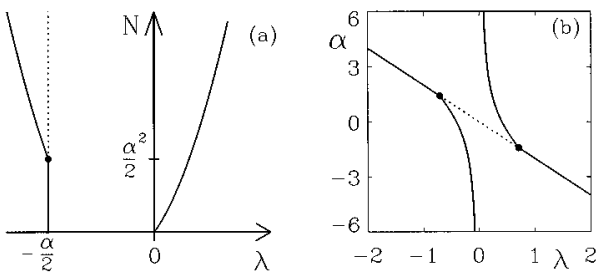

FIG. 2. (a) Norm $N$ as function of the eigenvalue $\lambda$ for the stationary solutions (I) and (II), given by Eqs. (3.3-3.4), with the phase-mismatch parameter $\alpha>0$. For $\alpha<0$ the curves are reversed around the vertical $N$ axis. (b) The dependence of $\alpha$ on $\lambda$ for $N=1$. Linearly stable (unstable) solutions are indicated by a solid (dotted) line.

which nonzero solutions do not exist. Such a gap is not found in the corresponding DST dimer, where there is a threshold instead [1]. Linear stability analysis shows that the solution (I) is stable for $N \leqslant \alpha^{2} / 2$, but unstable for $N>\alpha^{2} / 2$, while the solution (II) is stable in the whole domain of existence. From Fig. 2(b) we see that at fixed norm $N=1$ the solution (II) bifurcates continuously into the stable part of the solution (I) at $\alpha^{2}=4 \lambda^{2}=2$. Thus we have a stable stationary solution for all $\alpha$.

\section{B. Fixed points and dynamical solutions}

Let us introduce polar coordinates,

$$
W_{1}(\zeta)=\sqrt{x(\zeta)} e^{i \phi(\zeta)}, \quad V_{1}(\zeta)=\sqrt{y(\zeta)} e^{i \psi(\zeta)}
$$

where the intensities $x=N-2 y$ and $y$ are real and positive, and the phases $\phi$ and $\psi$ are real. Inserting Eq. (3.5) into Eqs. (3.1), and separating the real and imaginary parts, it is then straightforward to derive the Hamiltonian system of two conjugate degrees of freedom:

$$
\begin{gathered}
\dot{y}=-(N-2 y) \sqrt{y} \sin (\theta)=-\frac{\partial H}{\partial \theta}, \\
\dot{\theta}=\alpha+\left[\frac{6 y-N}{2 \sqrt{y}}\right] \cos (\theta)=\frac{\partial H}{\partial y},
\end{gathered}
$$

where $\theta(\zeta)=2 \phi(\zeta)-\psi(\zeta)$ and $H$ is given by

$$
H=\alpha y-(N-2 y) \sqrt{y} \cos (\theta) .
$$

Equations (3.6-3.7) assume that $y \neq 0$ and $y \neq N / 2$, and are therefore not physically valid in these limits, where the original Eqs. (3.1) must be applied instead.

Now let us look at the fixed points $(y, \theta)=\left(y_{e}, \theta_{e}\right)$, for which $\dot{y}=\dot{\theta}=0$. From Eq. (3.6) we see that there can be 4 such solutions, for which $y_{e}=0, y_{e}=N / 2$, and $\cos \left(\theta_{e}\right)= \pm 1$, respectively. Equations (3.1) give that the limit $y_{e}=0$ can never be a fixed point, while $y_{e}=N / 2$ always is a valid fixed point, since we can choose the phase $\phi(z)$ arbitrarily. In contrast, Eq. (3.7) gives unphysically that $y_{e}=N / 2$ is only valid for $|\alpha| \leqslant \sqrt{2}$ and $\cos \left(\theta_{e}\right)=-\alpha / \sqrt{2 N}$. The physically valid fixed points are therefore
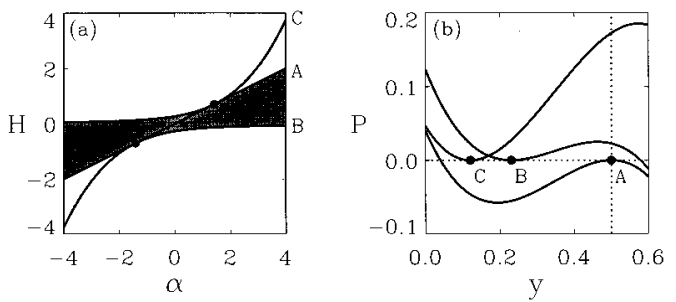

FIG. 3. (a) Regions of the $(H, \alpha)$ plane for $N=1$, in which the cubic equation $P(y)=0$, with $P(y)$ given by Eq. (3.11), has 1 real root (white), and 3 real roots (shaded). On the solid lines 2 of the 3 real roots are equal and at the points all 3 roots are equal. The darker shaded region indicates the physically valid regime. (b) The potential $P(y)$ for the 3 solid lines in (a), with $\alpha=0.4$. The points indicate the fixed point solutions (A) $-(\mathrm{C})$, given by Eqs. (3.9).

$$
\begin{aligned}
& \text { (A) } y_{e}=N / 2, \\
& \text { (B) } \cos \left(\theta_{e}\right)=+1, \quad \sqrt{y_{e}}=\left(\sqrt{\alpha^{2}+6 N}-\alpha\right) / 6, \\
& \text { (C) } \cos \left(\theta_{e}\right)=-1, \quad \sqrt{y_{e}}=\left(\sqrt{\alpha^{2}+6 N}+\alpha\right) / 6,
\end{aligned}
$$

where solution (A) exist for all $\alpha$, while (B) and (C) exists for $\alpha \geqslant-\sqrt{2 N}$ and $\alpha \leqslant \sqrt{2 N}$, respectively. The solutions (A) and (B) correspond to the stationary solutions (I) and (II), respectively (thus the stationary solutions are only a special branch of the fixed point solutions). Accordingly, we find that the solution (A) is linearly stable for $|\alpha| \geqslant \sqrt{2 N}$, and unstable for $|\alpha|<\sqrt{2 N}$, while the solutions (B) and (C) are stable in the whole domain of existence. The fixed points and their linear stability properties were found by Trillo et al., who studied second harmonic generation using phase-plane methods [30]. Here we will give a brief description of the analytical solution to the monomer. This will be useful for the discussion in Sec. IV, where we show that for certain initial conditions the dimer reduces to the monomer.

Equations (3.6-3.7) can be reduced to a single equation for the fraction of power in the second harmonic, $y(\zeta)$,

$$
\dot{y}^{2}+P(y)=0,
$$

which is equivalent to the dynamical equation for a classical particle moving in a potential $P(y)$. The potential $P(y)$ is a cubic polynomium,

$$
P(y)=-4 y^{3}+\left(\alpha^{2}+4 N\right) y^{2}-\left(2 \alpha H+N^{2}\right) y+H^{2} .
$$

In Fig. 3(a) we depict the number of real roots of the cubic equation $P=0$ in the $(H, \alpha)$ plane, which is simply given by the sign of the discriminant

$$
D=(H-\alpha N / 2)^{2}\left(H^{2}-d_{1} H-d_{0}\right) / 64,
$$

where $d_{0}=N^{2}\left(\alpha^{2}+8 N\right) / 108$ and $d_{1}=\alpha\left(\alpha^{2}+9 N\right) / 27$. Thus $P=0$ has 1 real root for $D>0$ (white regions) and 3 real roots for $D \leqslant 0$ (shaded regions), with at least two of them being equal for $D=0$ (solid lines). On the solid lines $H$ equals that of the fixed-point solutions (A), (B), and (C). The dark shaded region indicates the physically valid regime, in which $H(\alpha)$ can be obtained for $0<y<N / 2$ and $|\cos (\theta)| \leqslant 1$. At the two points $(H, \alpha)= \pm(1 / \sqrt{2}, \sqrt{2})$ there is a triple real root. 
The potential $P(y)$ is shown in Fig. 3(b) for $\alpha=0.4$ and $H$ on the 3 solid lines [fixed points (A), (B), and (C)]. From the particle analogy we can clearly identify the fixed points and their stability [(A) is unstable, (B) and (C) are stable].

In the physical region of the $(H, \alpha)$ plane, the general solution $y(\zeta)$ to Eq. (3.10) is a periodic function determined by the three real roots $0 \leqslant y_{1} \leqslant y_{2} \leqslant y_{3} \leqslant N / 2$,

$$
y(\zeta)=y_{1}+\left(y_{2}-y_{1}\right) \operatorname{sn}^{2}\left(\sqrt{y_{3}-y_{1}}\left[\zeta+\zeta_{0}\right], k\right)
$$

which oscillates between the two lowest roots $y_{1}$ and $y_{2}$. Here $\operatorname{sn}(u, k)$ is the Jacobi elliptic function with modulus $k^{2}=\left(y_{2}-y_{1}\right) /\left(y_{3}-y_{1}\right)$. This solution, where $\zeta_{0}$ is determined by the initial condition, was first found and studied by Armstrong et al. [14].

On the line $H=\alpha N / 2$, the two largest roots are identical to the fixed point (A), $y_{3}=y_{2}=N / 2\left(k^{2}=1\right)$, and $y_{1}=\alpha^{2} / 4$. In this case the period goes to infinity and the solution (3.13) reduces to

$$
y(\zeta)=\frac{1}{4} \alpha^{2}+\frac{1}{4}\left(2 N-\alpha^{2}\right) \tanh ^{2}\left(\frac{1}{2} \sqrt{2 N-\alpha^{2}}\left[\zeta+\zeta_{0}\right]\right) .
$$

This solution was first studied in [31]. It is of significant physical importance, since it predicts that total transfer of power to the second harmonic $(y \rightarrow N / 2)$ is possible. In the other simple cases, corresponding to the fixed points $(\mathrm{B})$ and (C), the two lowest roots are identical, $y_{1}=y_{2}(k=0)$, and the solution is a constant, $y(\zeta)=y(0)$. Thus no conversion to the second harmonic can be obtained.

\section{The Hamiltonian structure}

The quadratic monomer (3.1) has four degrees of freedom (amplitude and phase of $W_{1}$ and $V_{1}$ ). Since it has two conserved quantities and consists of two equations, it is completely integrable. Its solution in terms of the power $y$ in the second harmonic is well known [14] and has been reviewed in Sec. III B. In this section we give the Hamiltonian structure and underlying symmetry. From this the full solution, including the individual phases, can be found systematically.

For one waveguide the Hamiltonian (2.6) reduces to

$$
H=\alpha\left|V_{1}\right|^{2}-\operatorname{Re}\left\{W_{1}^{2} V_{1}^{*}\right\},
$$

where $W_{1}$ and $V_{1}$ satisfy the Poisson structure $\left\{W_{1}, i W_{1}^{*}\right\}=1,\left\{V_{1}, i V_{1}^{*}\right\}=1$, with the Poisson brackets being defined as

$$
\{a, b\} \equiv-i \sum_{n=1}^{n_{0}} \sum_{j=1}^{2} \frac{\partial a}{\partial \phi_{j n}} \frac{\partial b}{\partial \phi_{j n}^{*}}-\frac{\partial b}{\partial \phi_{j n}} \frac{\partial a}{\partial \phi_{j n}^{*}},
$$

where $\phi_{1 n}=W_{n}$ and $\phi_{2 n}=V_{n}$. Thus $\left(W_{1}, i W_{1}^{*}\right)$ and $\left(V_{1}, i V_{1}^{*}\right)$ are Hamiltonian conjugate variables. Using the Poisson structure, the equations of motion (3.1) can be written as $\dot{W}_{1}=\left\{W_{1}, H\right\}$ and $\dot{V}_{1}=\left\{V_{1}, H\right\}$.

Consider the compact su(2) algebra

$$
\begin{gathered}
\left\{r_{1}, r_{2}\right\}=r_{3}, \quad\left\{r_{2}, r_{3}\right\}=r_{1}, \quad\left\{r_{3}, r_{1}\right\}=r_{2}, \\
\left\{C, r_{j}\right\}=0, \quad j=1,2,3, \quad C=r_{1}^{2}+r_{2}^{2}+r_{3}^{2},
\end{gathered}
$$

where $C$ acts as the Casimir element. Because of the symmetric group structure, the conjugate variables $p_{j}$, $\left\{r_{j}, p_{j}\right\}=1$, are given by

$$
\begin{gathered}
p_{1}=\arctan \left(r_{2} / r_{3}\right), \quad p_{2}=\arctan \left(r_{3} / r_{1}\right), \\
p_{3}=\arctan \left(r_{1} / r_{2}\right) .
\end{gathered}
$$

For Eqs. (3.1) the algebra (3.16) can be realized by the Feynman variables

$$
\begin{gathered}
r_{1}=\sqrt{2\left|V_{1}\right|} \operatorname{Re}\left\{W_{1}^{*} \sqrt{V_{1}}\right\}, \\
r_{2}=\sqrt{2\left|V_{1}\right|} \operatorname{Im}\left\{W_{1}^{*} \sqrt{V_{1}}\right\}, \\
r_{3}=\left(\left|W_{1}\right|^{2}-2\left|V_{1}\right|^{2}\right) / 2, \\
C=\left(\left|W_{1}\right|^{2}+2\left|V_{1}\right|^{2}\right) / 2,
\end{gathered}
$$

in terms of which the Hamiltonian may be written as

$$
H=\alpha\left(C-r_{3}\right) / 2+\left(r_{2}^{2}-r_{1}^{2}\right) / \sqrt{C-r_{3}} .
$$

The Casimir element is proportional to the norm, $C=N / 2$, and therefore a constant of motion, $\dot{C}=\{C, H\}=0$. Thus the monomer (3.1) has a simple su(2) group structure, closely related to its integrability. The integrable DST dimer has a similar structure [28].

The system is considerably simplified if we introduce polar coordinates as in Eq. (3.4):

$$
\begin{aligned}
& W_{1}(\zeta)=\sqrt{x(\zeta)} e^{i[\sigma(\zeta)+\delta(\zeta)] / 4} \\
& V_{1}(\zeta)=\sqrt{y(\zeta)} e^{i[\sigma(\zeta)-\delta(\zeta)] / 2}
\end{aligned}
$$

where we have written the phases in terms of the sum $\sigma=$ $(2 \phi+\psi) / 2$ and the difference $\delta=(2 \phi-\psi) / 2$. Since $\{C, \sigma\}=1$ we see that $\sigma$ has no influence on the dynamics of the other variables, and thus is an ignorable coordinate, as we would expect from the calculations in Sec. III B, where only $\theta=2 \delta$ appears in Eqs. (3.6-3.7). In polar coordinates the Feynman variables simplify to

$$
\begin{gathered}
r_{1}=\sqrt{2 x y} \cos (\delta), \quad r_{2}=-\sqrt{2 x y} \sin (\delta), \\
r_{3}=(x-2 y) / 2, \quad C=(x+2 y) / 2,
\end{gathered}
$$

where $\delta$ is the conjugate variable of $r_{3},\left\{r_{3}, \delta\right\}=1$.

Introducing a new evolution coordinate $d \xi=r_{1} r_{2} d \zeta$ the dynamical equations $\dot{r}_{j}=\left\{r_{j}, H\right\}$ become

$$
r_{1,2}^{\prime}= \pm \frac{3 \alpha}{4} \mp \frac{H}{2\left(C-r_{3}\right)}+r_{3} \sqrt{\frac{2}{C-r_{3}}}
$$

$$
r_{3}^{\prime}=-2 \sqrt{2 /\left(C-r_{3}\right)}
$$

where a prime denotes differentiation with respect to the new coordinate $\xi$. Integrating the equation for $r_{3}$ and subse- 
quently the equations for $r_{1}$ and $r_{2}$, we obtain Eqs. (3.6-3.7), where $y=\left[3\left(\xi-\xi_{0}\right) / 3\right]^{2 / 3}, \xi_{0}$ being an integration constant.

The dynamical equations for $\delta$ and for the ignorable coordinate $\sigma$ are obtained as $\dot{\delta}=\{\delta, H\}$ and $\dot{\sigma}=\{\sigma, H\}$, thus yielding the dynamics of the individual phases $\phi$ and $\psi$. This is of crucial importance for, e.g., Mach-Zehnder interferometers, but it is only recently that this calculation has been carried out in detail [32], without considering the Hamiltonian $\operatorname{su}(2)$ structure though.

\section{TWO WAVEGUIDES - THE DIMER}

We now consider two waveguides and thus the system (2.3-2.4) reduces to the four coupled equations

$$
\begin{gathered}
i \dot{W}_{n}+W_{3-n}+W_{n}^{*} V_{n}=0, \\
i \dot{V}_{n}+\eta V_{3-n}-\alpha V_{n}+W_{n}^{2} / 2=0, \quad n=1,2,
\end{gathered}
$$

which are not integrable, just as the DST equation for four sites [1]. However, we can still find all stationary solutions analytically and check their stability, which we do in Sec. IV A. We find 7 different classes of solutions, two of which have been found before [12], however, without considering the stability and the general scaling invariance given by Eqs. (2.7). In Sec. IV B we discuss the self-trapping properties of the stationary solutions. In particular we show how the stationary solutions are simply connected to the known stationary solutions of the DST dimer in the limit $|\alpha| \gg 1$. Furthermore, we use the self-trapped DST stationary solutions as initial condition in the full quadratic system, and show how their dynamics gradually becomes more chaotic as $|\alpha|$ is decreased. Finally we discuss the Hamiltonian structure of the equations in Sec. IV C and show how they can be reduced to a Hamiltonian system of six degrees of freedom.

\section{A. Stationary solutions}

In analogy with the monomer we consider stationary solutions of the form

$$
W_{n}(\zeta)=w_{n} e^{i \lambda \zeta}, \quad V_{n}(\zeta)=v_{n} e^{i 2 \lambda \zeta}
$$

where $\lambda$ is real and $w_{1}$ is real and non-negative. All solutions with $w_{1}$ complex may be obtained from the phase rotation invariance given by Eq. (2.7). For two waveguides Eqs. (2.32.4) then reduce to

$$
\begin{gathered}
{\left[\begin{array}{cc}
\lambda & -1 \\
-1 & \lambda
\end{array}\right]\left[\begin{array}{l}
w_{1} \\
w_{2}
\end{array}\right]=\left[\begin{array}{l}
w_{1} v_{1} \\
w_{2}^{*} v_{2}
\end{array}\right],} \\
{\left[\begin{array}{cc}
\alpha+2 \lambda & -\eta \\
-\eta & \alpha+2 \lambda
\end{array}\right]\left[\begin{array}{l}
v_{1} \\
v_{2}
\end{array}\right]=\left[\begin{array}{l}
w_{1}^{2} / 2 \\
w_{2}^{2} / 2
\end{array}\right] .}
\end{gathered}
$$

Here Eq. (4.5) can be considered as a linear inhomogeneous set of equations, with the determinant $D=4\left(\lambda-\lambda_{+}\right)\left(\lambda-\lambda_{-}\right)$, where $\lambda_{ \pm} \equiv(-\alpha \pm \eta) / 2$. For $D=0$ there exist three qualitatively different solutions

$$
\begin{aligned}
& w_{n}=0, \quad\left|v_{1}\right|^{2}+\left|v_{2}\right|^{2}=N / 2 \text { for } \lambda=\lambda_{ \pm}, \\
& w_{2}= \pm w_{1}, \quad v_{n}=\lambda \mp 1 \text { for } \lambda=\lambda_{-}, \quad \eta \neq 0, \\
& w_{2}= \pm i w_{1}, \quad v_{1}=\lambda \mp i, \quad v_{2}=-\lambda \mp i \\
& \text { for } \lambda=\lambda_{+}, \quad \eta \neq 0,
\end{aligned}
$$

where $w_{1}=\sqrt{N / 2-2\left|v_{1}\right|^{2}}$ in solutions (II) and (III). The solution (I) is equivalent to that for the monomer. For $D \neq 0$ Eq. (4.5) gives the second harmonic

$$
v_{n}=\left[(\alpha+2 \lambda) w_{n}^{2}+\eta w_{3-n}^{2}\right] /(2 D),
$$

in terms of the fundamental. In this case there exist four classes of solutions. When $w_{2}^{2}=w_{1}^{2}$ the solution is

$$
\begin{aligned}
& \text { (IV) } w_{2}= \pm w_{1}= \pm \sqrt{4(\lambda \mp 1)\left(\lambda-\lambda_{+}\right)} \text {, } \\
& v_{n}=\lambda \mp 1 \text { for } D \neq 0, \quad(\lambda \mp 1)\left(\lambda-\lambda_{+}\right)>0 \text {. }
\end{aligned}
$$

When $w_{2}$ is allowed to be complex, and $\left|w_{2}\right|^{2}=w_{1}^{2}$, the fundamental fields are found to

$$
\begin{array}{ll}
\text { (V) } & w_{1}=\sqrt{4 \lambda\left(\lambda-\lambda_{-}\right)}, \\
& w_{2}=-D /\left(\eta w_{1}\right) \pm i \sqrt{w_{1}^{2}-D^{2} /\left(\eta w_{1}\right)^{2}} \\
& \text { for } D \neq 0, \quad \lambda\left(\lambda-\lambda_{-}\right)>0, \quad \eta \neq 0, \\
& \eta^{2} \lambda^{2}>\left(\lambda-\lambda_{+}\right)^{2},
\end{array}
$$

with $v_{n}$ being given by Eq. (4.7). In the general case when $\left|w_{2}\right|^{2} \neq w_{1}^{2}$, there are two solutions, for which the fundamentals are given by

$$
\begin{aligned}
& w_{2}=4\left(\lambda-\lambda_{-}\right) / w_{1}, \quad w_{1}=\sqrt{x_{1}} \quad \text { or } \sqrt{x_{2}} \\
& \text { for } D \neq 0, \quad \lambda \neq-\alpha / 2, \quad b<-2 \sqrt{c},
\end{aligned}
$$

where $x_{n}$ are the two roots of the quadratic equation

$$
\begin{gathered}
x^{2}+b x+c=0, \\
b=-2 \lambda D /(\alpha+2 \lambda), \quad c=16\left(\lambda-\lambda_{-}\right)^{2},
\end{gathered}
$$

and $v_{n}$ is given by Eq. (4.7). Finally, in the special case when $\lambda=-\alpha / 2$ and $\left|w_{2}\right|^{2} \neq w_{1}^{2}$, the solution is

$$
\begin{aligned}
& \text { (VII) } w_{2}=\frac{2 \eta}{w_{1}}, \quad v_{1}=-\frac{2 \eta}{w_{1}^{2}}, \quad v_{2}=-\frac{w_{1}^{2}}{2 \eta} \\
& \text { for } D \neq 0, \quad \lambda=\alpha=0, \quad w_{1} \neq 0, \quad \eta \neq 0,
\end{aligned}
$$

where $w_{1}$ can be found from $N$. In the following we will denote the solutions (IV), (V), and (VI) as general solutions, because they exist in a certain interval in $\lambda$, while the solutions (I), (II), (III), and (VII) are termed particular solutions, because they only exist for discrete values of $\lambda$.

Due to the scaling invariance, given by Eq. (2.7), each of the classes (I)-(VII) correspond to infinitely many solutions. The symmetric (IV) + and antisymmetric (IV) _ solutions, as 

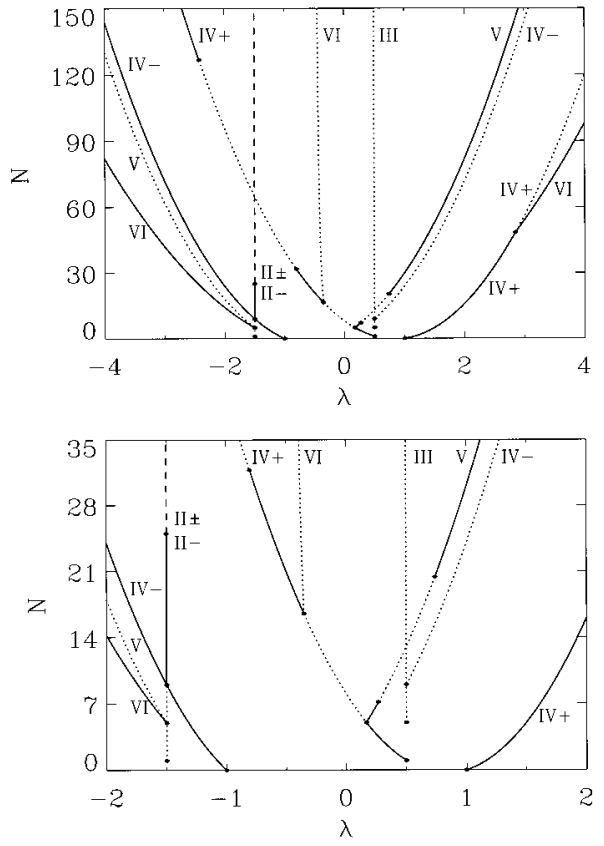

FIG. 4. Norm $N$ as a function of the eigenvalue $\lambda$ for the stationary solutions (II) $-(\mathrm{VII})$, with $\eta=2 \alpha=2$. Linearly stable (unstable) solutions are indicated by a solid (dotted) line and the curves are labeled with the solution they represent. The dashed line indicates that the (II) $+\left[\left(\mathrm{II}_{-}\right)\right]$solution is unstable (stable). The bottom figure shows a closeup for small $N$.

well as the (VI) solution, were also found by Dubovskii and Orlov [12]. However, the issue of stability was not considered, and only the sign degeneracy of the fundamental $[\Omega=$ $\pi$ in Eq. (2.7)] was taken into account.

In Fig. 4 we show the norm $N$ as a function of the soliton eigenvalue $\lambda$ for the solutions (II)-(VII). As a representative example we have used $\eta=2 \alpha=2$, for which solution (VII) does not exist. Stable (unstable) solutions are indicated by a solid (dotted) line. The solutions (II), (III), (V), and (VI) all have two degenerate branches, which cannot be distinguished by this $N(\lambda)$ diagram. In Fig. 4 these pair of solutions all have the same stability properties, except for the solution (II), for which the component with the plus (minus) sign is unstable (stable) for $N>25$, and does not exist for lower values of $N$.

The solution (I) is special, since for each value of the norm, there exist infinitely many subbranches of solutions, whose stability properties vary with the relative size of $\left|v_{1}\right|^{2}$ and $\left|v_{2}\right|^{2}$. In Fig. 5 we show the stability regimes of the solution (I) as a function of $2\left|v_{1}\right|^{2} / N \in[0,1]$ for $\eta=2 \alpha=2$, as in Fig. 4. The curve that separates the stable and unstable regimes is shown as a solid line for the branch at $\lambda=\lambda_{+}$and a dashed line for the branch at $\lambda=\lambda_{-}$. For both branches the solution is unstable if it has a large value of the norm, but becomes stable for sufficiently small values of the norm.

\section{B. Self-trapping and the DST limit}

The dynamical equations (4.1-4.2) for 2 waveguides are nonintegrable, and consequently the dynamics may be chaotic. However, the stationary solutions constitute important points in the phase space, around which the system evolves.

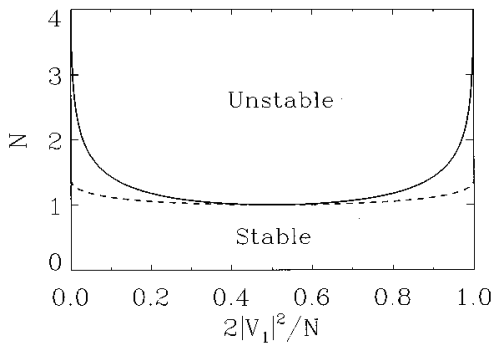

FIG. 5. Norm $N$ vs normalized power $2\left|v_{1}\right|^{2} / N$ for the stationary solution (I) at $\lambda=\lambda_{+}$(solid) and $\lambda=\lambda_{-}$(dashed), with $\eta=2 \alpha=2$. In the regime below (above) the curve the solution is linearly stable (unstable).

Of particular interest are the self-trapped states, where the norm is primarily concentrated in one of the degrees of freedom. Here we will consider trapping in terms of whether the norm is concentrated in one of the two waveguides, $N \approx N_{n}=$ $\left|W_{n}\right|^{2}+2\left|V_{n}\right|^{2}$.

In Fig. 6 we therefore depict the ratio $N_{1} / N_{2}$ of the norm in the two waveguides, for the stationary solutions (I)-(VII). The parameters are the same as in Figs. 4 and 5, $\eta=2$ and $\alpha=1$, and thus the particular solution (VII) does not exist. We see that of the general solutions (IV), (V), and (VI), only the solution (VI), for which $\left|W_{1}\right|^{2} \neq\left|W_{2}\right|^{2}$, can correspond to a self-trapped state, with either $N_{1} \approx N$ or $N_{2} \approx N$. Of the particular solutions (I), (II), (III), and (VII), only the solution (I) can represent a self-trapped state. Note that with this representation the two degenerate (VI) solutions, $(\mathrm{VI})_{+}$and (VI) ${ }_{-}$, can be clearly distinguished.

Let us consider the limit where $|\alpha| \rightarrow \infty$, while both $\dot{V}_{n}$ and the coupling parameter $\eta$ remain finite. Eqs. (4.1-4.2) can then be reduced to

$$
i \dot{W}_{n}+W_{3-n}+\gamma\left|W_{n}\right|^{2} W_{n}=0, \quad n=1,2
$$

where the nonlinearity parameter $\gamma=1 /(2 \alpha)$ and the second harmonic is given by $V_{n}=\gamma W_{n}^{2}$. The expressions for the general solutions (IV) and (VI) in this limit, where $|\alpha| \gg|\lambda|$ and $|\alpha| \gg|\eta|$, are given in Table I, from which it is clearly seen that the solutions $\mathrm{VI}_{ \pm}$correspond to a self-trapped state for sufficiently large values of $|\lambda|$. All other solutions do not exist in this limit.

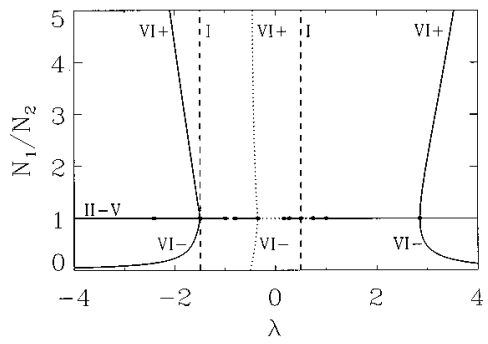

FIG. 6. Ratio of the norm in the two waveguides, $N_{n}=$ $\left|W_{n}\right|^{2}+2\left|V_{n}\right|^{2}$, vs the eigenvalue $\lambda$ for $\eta=2 \alpha=2$. Linearly stable (unstable) solutions are indicated by a solid (dotted) line. The dashed line indicates the (I) solution, which can be stable or unstable, depending on the total norm $N$. 
TABLE I. The fundamental $W_{n}(\zeta)=w_{n} \mathrm{e}^{i \lambda \zeta}$ for the stationary solutions $\mathrm{IV}_{ \pm}$and $\mathrm{VI}_{ \pm}$in the limit where $|\alpha| \rightarrow \infty$, while $|\alpha| \gg|\lambda|$ and $|\alpha| \gg|\eta|$. The second harmonic is $V_{n}=\gamma W_{n}^{2}$.

\begin{tabular}{lcc}
\hline \hline Solution & Fundamental & Requirement \\
\hline $\mathrm{IV}_{ \pm}$ & $w_{1}= \pm w_{2}=\sqrt{2 \alpha(\lambda \mp 1)}$ & $\alpha(\lambda \mp 1)>0$ \\
$\mathrm{VI}_{ \pm}$ & $w_{1}=\sqrt{\lambda \alpha\left[1 \pm \sqrt{1-4 / \lambda^{2}}\right]}$ & $\lambda \operatorname{sign}\{\alpha\}>2$ \\
& $w_{2}=\sqrt{\lambda \alpha\left[1 \mp \sqrt{1-4 / \lambda^{2}}\right]}$ & \\
\hline \hline
\end{tabular}

Equation (4.13) is also known as the DST equation [1] or discrete NLS equation with two degrees of freedom [9]. The stationary solutions of this integrable dimer were first studied by Eilbeck et al., who found four different solutions [1]. Comparing Table I with Table I in [1] (with $\epsilon=1$ ) we see that $\left(W_{1}, W_{2}\right)$ for the asymptotic stationary solutions (IV) ${ }_{+}$, $(\mathrm{IV})_{-},(\mathrm{VI})_{+}$, and (VI) ${ }_{-}$to the quadratic Eqs. (4.1-4.2), correspond exactly to the DST stationary solutions termed $\uparrow \uparrow, \uparrow \downarrow, \uparrow \cdot$, and $\cdot \uparrow$, respectively. The close connection with the integrable DST dimer when $|\alpha| \rightarrow \infty$ indicates that the quadratic dimer is near integrable in this limit. An interesting question is therefore how the transition from integrability to nonintegrability occurs when decreasing $|\alpha|$. This can be illustrated by looking at how the DST-like stationary solutions, given in Table I, behave when used as inital condition in Eqs. (4.1-4.2), for decreasing values of $|\alpha|$.

In Fig. 7 we show the results of numerical integration of Eqs. (4.1-4.2) with the solution (VI) + as initial condition, for $\lambda=2.5, \eta=1$, and $\alpha=100,50,41$, and 38. The dynamics is illustrated as a phase-plane plot showing $M \equiv\left(\left|W_{1}\right|^{2}-\left|W_{2}\right|^{2}\right) / N_{w}$ versus $\Theta \equiv \operatorname{Arg}\left\{W_{1} / W_{2}\right\}$, where $N_{w}=\left|W_{1}\right|^{2}+\left|W_{2}\right|^{2}$ is the norm of the fundamental. For $|\alpha| \rightarrow \infty$, where the solution corresponds to a trapped stationary DST solution with most of the norm $N_{w}=2 \lambda \alpha$ at site 1 , the trajectory is simply a point at $(M, \Theta)=(0,0.6)$, indicated

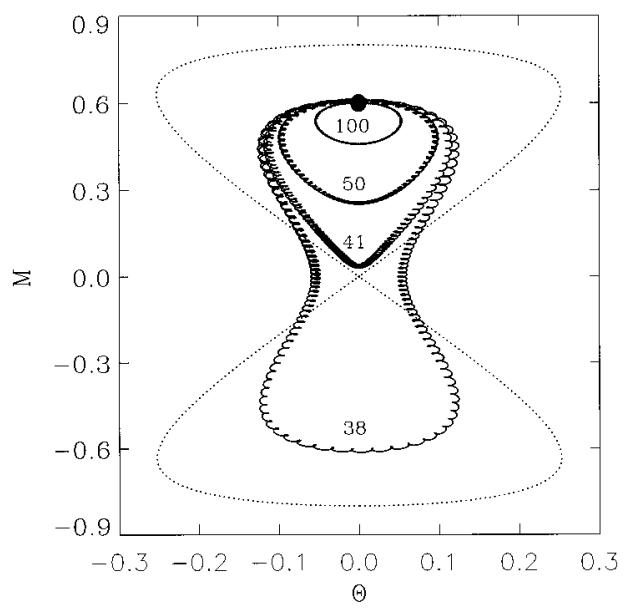

FIG. 7. $M$ vs $\Theta$ for the dynamics of the asymptotic (VI) + solution given in Table I, when evolving in the system (4.1-2). The parameters are $\eta=1$ and $\lambda=2.5$, with $\alpha$ being given at the different curves. In all cases the integration distance was $z=20$. The dotted line indicates the DST separatrix $\left(M_{s}, \Theta_{s}\right)$ (4.14). The filled circle indicates the point $(M, \Theta)=(0,0.6)$, corresponding to the limit $\alpha \rightarrow \infty$.
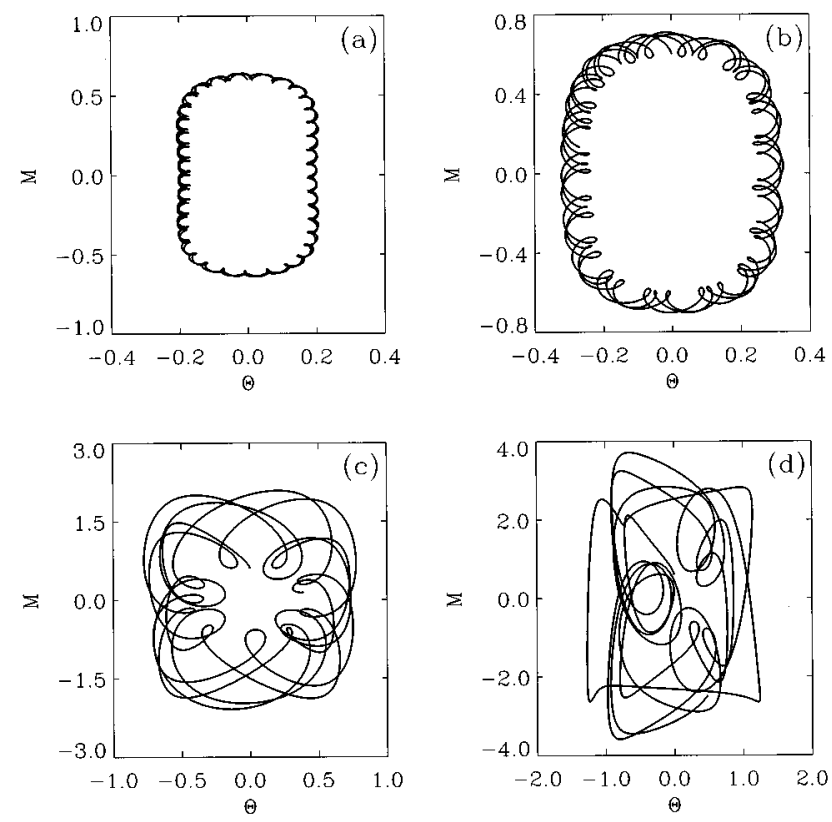

FIG. 8. $M$ vs $\Theta$ for the dynamics of the asymptotic $(\mathrm{VI})_{+}$ solution given in Table I, when evolving in the system (4.1-4.2). The parameters are $\eta=1, \lambda=2.5$, and $\alpha=20$ (a), 10 (b), 1 (c), and 0.4 (c). The integration distance is $z=20$.

by a filled circle. This is also the initial point of integration. The dotted line indicates the DST separatrix, separating trapped (inside) from nontrapped (outside) solutions to the DST equation (4.13). In terms of $\gamma$ and the norm $N$ this separatrix is given by

$$
M_{s}=\frac{2}{N} N_{1}-1, \quad \cos \left(\Theta_{s}\right)=\frac{1-\gamma N M^{2} / 4}{\sqrt{1-M^{2}}},
$$

where $N_{1}(z)= \pm a \operatorname{sech}(\gamma a z)$, with $a=\sqrt{2 \gamma N-4} / \gamma$. We see that the coupling to the second harmonic due to a finite but large $\alpha$ introduces regular oscillations that grow when $\alpha$ is decreased. For $\alpha>40.9$ the difference $\left|W_{1}\right|^{2}-\left|W_{2}\right|^{2}$ is always positive. Thus most of the fundamental power remains at site 1 , and the solution can still be classified a trapped solution. For $\alpha<40.9$ the trajectory crosses the DST separatrix, which makes the fundamental power swop periodically between the two sites. Thus the solution is no longer trapped. However, the motion is still regular, even for $\alpha=38$.

In Fig. 8 we show the trajectories for even smaller $\alpha$ values, $\alpha=20,10,1$, and 0.4 . The motion becomes more and more chaotic as $\alpha$ is decreased, with almost complete chaos for $\alpha=0.4$. However, even for $\alpha=10$, the motion is still highly regular and symmetric, showing a periodic exchange of fundamental power between the two sites. We have found that this gradual transition to chaos as $\alpha$ is decreased is generally representative for the behavior of the asymptotic solutions $(\mathrm{VI})_{ \pm}$in Table I, that correspond to stable self-trapped states in the DST limit $|\alpha| \rightarrow \infty$.

In order to see the corresponding effect on the delocalized asymptotic solutions (IV) $)_{ \pm}$, we do not need to perform numerical simulations. For any value of $\alpha$ the initial condition $W_{1}= \pm W_{2}=W$, and $V_{1}=V_{2}=V$ belong to a special class of "integrable initial conditions," which reduces the dimer (4.1-4.2) to the integrable monomer 


$$
i \dot{\widetilde{W}}+\widetilde{W}^{*} \widetilde{V}=0, \quad i \dot{\bar{V}}-\widetilde{\alpha} \widetilde{V}+\widetilde{W}^{2} / 2=0,
$$

after the transformation

$$
W=\widetilde{W} e^{\mp i z}, \quad V=\widetilde{V} e^{\mp i 2 z}, \quad \alpha=\widetilde{\alpha}+\eta \pm 2 .
$$

Thus the motion of the two sites is completely decoupled, and the individual power $N_{n}$ at each site $n$ is conserved. Some part of the power will simply oscillate periodically between the fundamental $W_{n}$ and second harmonic $V_{n}$, as given by Eq. (3.13). Changing $\alpha$ only leads to a change of the three roots $y_{1}, y_{2}$, and $y_{3}$, and thus the amplitude and frequency of the oscillations.

For the symmetric initial condition $W_{n}=W$, and $V_{n}=V$, the full system (2.3-2.4) can be reduced to the monomer (3.1), regardless of the number of sites in the system. Such "integrable initial conditions" are also found in the DST equation (4.13) for $W_{n}=W$.

\section{The Hamiltonian structure}

The dimer, given by Eqs. (4.1-4.2), is nonintegrable, and obviously cannot be written as a simple su(2) algebra, as is the case for the integrable monomer. However, we can still reduce the system of 8 degrees of freedom to a Hamiltonian system of 6 degrees of freedom, which has a close resemblance with the monomer. To do so we introduce polar coordinates as in Eq. (3.4),

$$
W_{j}(\zeta)=\sqrt{x_{j}(\zeta)} e^{i \phi_{j}(\zeta)}, \quad V_{j}(\zeta)=\sqrt{y_{j}(\zeta)} e^{i \psi_{j}(\zeta)}
$$

and note that the phases can be written as

$$
\begin{gathered}
\phi_{1}=\left(\frac{\delta}{4}+\frac{\phi}{2}\right)+\frac{\sigma}{4}, \quad \phi_{2}=\left(\frac{\delta}{4}-\frac{\phi}{2}\right)+\frac{\sigma}{4}, \\
\psi_{1}=\left(-\frac{\delta}{2}+\frac{\psi}{2}\right)+\frac{\sigma}{2}, \quad \psi_{2}=\left(-\frac{\delta}{2}-\frac{\psi}{2}\right)+\frac{\sigma}{2},
\end{gathered}
$$

where the collective phases $\delta, \phi, \psi$, and $\sigma$ are defined as

$$
\begin{aligned}
& \delta=\left(2\left[\phi_{1}+\phi_{2}\right]-\left[\psi_{1}+\psi_{2}\right]\right) / 2, \quad \phi=\phi_{1}-\phi_{2}, \\
& \sigma=\left(2\left[\phi_{1}+\phi_{2}\right]+\left[\psi_{1}+\psi_{2}\right]\right) / 2, \quad \psi=\psi_{1}-\psi_{2} .
\end{aligned}
$$

From the scaling property, given by Eq. (2.7), we see that the sum $\sigma$ is an ignorable coordinate, just as the equivalent sum for the monomer. The conjugate variables to the remaining three phases are

$$
\begin{gathered}
R_{1}=\left(x_{1}-x_{2}\right) / 2, \quad R_{2}=\left(y_{1}-y_{2}\right) / 2, \\
R_{3}=\left(\left[x_{1}+x_{2}\right]-2\left[y_{1}+y_{2}\right]\right) / 4,
\end{gathered}
$$

where $\left\{R_{1}, \phi\right\}=1,\left\{R_{2}, \psi\right\}=1$, and $\left\{R_{3}, \delta\right\}=1$, with the Poisson bracket being defined in Eq. (3.15). However, the $R_{j}$ 's do not satisfy the group structure given by Eq. (3.16), and $R_{1}^{2}+R_{2}^{2}+R_{3}^{2}$ is not a conserved quantity.

The individual intensities $x_{j}$ and $y_{j}$ may easily be written in terms of the $R_{j}$ 's and the norm. Thus the six conjugate variables $\left(R_{1}, \phi\right),\left(R_{2}, \psi\right)$, and $\left(R_{3}, \delta\right)$ comprises the reduced system, in which the Hamiltonian is given by

$$
\begin{aligned}
H= & \alpha N_{-}-2 \sqrt{N_{+}^{2}-R_{1}^{2}} \cos (\phi)-\eta \sqrt{N_{-}^{2}-4 R_{2}^{2}} \cos (\psi) \\
& -\left(N_{+}+R_{1}\right) \sqrt{\frac{1}{2} N_{-}+R_{2}} \cos \left(\delta+\phi-\frac{1}{2} \psi\right) \\
& -\left(N_{+}-R_{1}\right) \sqrt{\frac{1}{2} N_{-}-R_{2}} \cos \left(\delta-\phi+\frac{1}{2} \psi\right),
\end{aligned}
$$

where $N_{ \pm}=N / 4 \pm R_{3}$. The six dynamical equations may be obtained as Hamilton equations, but these are far more complicated than Eqs. (4.1-4.2), which is why we have used Eqs. (4.1-4.2) when integrating the dimer numerically. However, the equations can throw some light on the structure of the problem, which may be useful for future research on the general system (2.3-2.4).

\section{DISCUSSION}

In this work we have studied the generic model for coupled quadratic nonlinear oscillators, each having two harmonic frequencies close to resonance. Being generic this model appears as the basic model in many areas of fundamental physics and nonlinear science. Specific applications include waveguide arrays in quadratic nonlinear, so-called $\chi^{(2)}$ materials, and interface waves between two media close to Fermi resonance.

Large arrays of nonlinear waveguides are not relevant with the present technology. We have therefore studied the system with one and two coupled oscillators, the monomer and dimer, with special emphasis on the dimer, which has not yet been investigated in detail. In particular we have analytically found all stationary solutions, and determined their stability and self-trapping properties. Furthermore, we have analyzed the Hamiltonian structure of the systems.

The stationary modes are an important subclass of solutions for the characterization of a given system, and may constitute the starting point for further analysis of the dynamical properties. The essential parameter for the dynamics is the phase-mismatch parameter $\alpha$. When $|\alpha|$ is large the second harmonic mode is weak, and slaved to the fundamental mode, whose dynamics then determines the dynamics of the whole system. In this limit the dominant fundamental mode is described by the DST equation, which is the generic model for coupled cubic nonlinear oscillators with only a single frequency. In nonlinear optics this is well known for the corresponding continuum systems: Away from phase matching $\chi^{(2)}$ materials have effective cubic properties, in fact their nonintegrable dynamical equations reduce to the NLS equation, which is integrable in one dimension.

However, the discrete equations studied here are more intriguing than their continuum counterpart in that their integrability properties, and those of the effective equations in the large phase-mismatch limit, depend of the number of oscillators. For one oscillator both the original system and the limiting DST system are integrable. In the more interesting case of two oscillators, the $\chi^{(2)}$ dimer is not integrable, but reduces to the integrable DST dimer in the limit of a 
large phase mismatch. We have given the specific connection between the stationary solutions of these two systems in this near-integrable limit. Five of the seven classes of $\chi^{(2)}$ solutions disappear, while the two remaining becomes the localized (self-trapped) and delocalized DST solutions.

Finally, we have used the approximate self-trapped solution obtained in the near-integrable DST limit as an initial condition in the $\chi^{(2)}$ dimer, and numerically shown how this solution gradually develops dynamics. For large $|\alpha|$ it is almost stationary, while the dynamics becomes more and more chaotic as $|\alpha|$ is decreased and we approach exact phase matching. At a specific value of $\alpha$ there is a transition, where the solution goes from being primarily localized at one oscillator to being delocalized.

\section{ACKNOWLEDGMENTS}

We would like to acknowledge M.F. Jørgensen, J.C. Eilbeck, F. Lederer, U. Peschel, and C. Etrich for valuable suggestions, and the financial support of the Department of Industry, Science and Tourism, under the Bilateral Science and Technology Program, Grant No. 96/4579.
[1] J.C. Eilbeck, P.S. Lomdahl, and A.C. Scott, Physica D 16, 318 (1985).

[2] T. Holstein, Ann. Phys. (N.Y.) 8, 325 (1959).

[3] A.S. Davydov and N.I. Kislukha, Phys. Status Solidi B 59, 465 (1973).

[4] A.C. Scott and J.C. Eilbeck, Chem. Phys. Lett. 132, 23 (1986).

[5] J.C. Eilbeck, P.S. Lomdahl, and A.C. Scott, Phys. Rev. B 30, 4703 (1984).

[6] J.H. Jensen, P.L. Christiansen, O. Skovgaard, O.F. Nielsen, and I.J. Bigio, Phys. Lett. A 117, 123 (1986).

[7] H. Feddersen, Phys. Lett. A 154, 391 (1991).

[8] S.M. Jensen, IEEE J. Quantum Electron. 18, 1580 (1982).

[9] D.N. Christodoulides and R.I. Joseph, Opt. Lett. 13, 794 (1988).

[10] A.B. Aceves, C. De Angelis, T. Peschel, R. Muschall, F. Lederer, S. Trillo, and S. Wabnitz, Phys. Rev. E 53, 1172 (1996).

[11] V.M. Agranovich and O.A. Dubovsky, Chem. Phys. Lett. 210, 458 (1993); V.M. Agranovich and A.M. Kamchatnov, Pis'ma Zh. Éksp. Teor. Fiz. 59, 397 (1994) [JETP Lett. 59, 424 (1994)].

[12] S.A. Dubovskii and A.V. Orlov, Fiz. Tverd. Tela 38, 1221 (1996) [Phys. Solid State 38, 675 (1996)]; 38, 1931 (1996) [38, 1067 (1996)].

[13] T. Peschel, U. Peschel, and F. Lederer, Phys. Rev. E 55, 4730 (1997).

[14] J.A. Armstrong, N. Bloembergen, J. Ducuing, and P.S. Pershan, Phys. Rev. 127, 1918 (1962).

[15] G.I. Stegeman, E.M. Wright, N. Finlayson, R. Zanoni, and T. Seaton, IEEE J. Light. Technol. 6, 953 (1988).

[16] G.I. Stegeman, D.J. Hagan, and L. Torner, Opt. Quantum Electron. 28, 1691 (1996).

[17] V.E. Zakharov and A. B. Shabat, Zh. Éksp. Teor Fiz. 61, 118
(1971) [Sov. Phys. JETP 34, 62 (1972)].

[18] J.J. Rasmussen and K. Rypdal, Phys. Scr. 33, 481 (1986).

[19] L. Bergé, V.K. Mezentsev, J.J. Rasmussen, and J. Wyller, Phys. Rev. A 52, R28 (1995); L. Bergé, O. Bang, J.J. Rasmussen, and V.K. Mezentsev, Phys. Rev. E 55, 3555 (1997).

[20] A.A. Kanashov and A.M. Rubenshik, Physica D 4, 122 (1981); L. Torner, C.R. Menyuk, W.E. Torruellas, and G.I. Stegeman, Opt. Lett. 20, 13 (1995).

[21] D.E. Pelinovsky, A.V. Buryak, and Y.S. Kivshar, Phys. Rev. Lett. 75, 591 (1995).

[22] W.E. Torruellas, Z. Wang, D.J. Hagan, E.W. Van Stryland, G.I. Stegeman, L. Torner, and C.R. Menyuk, Phys. Rev. Lett. 74, 5036 (1995).

[23] R. Schiek, Y. Baek, and G.I. Stegeman, Phys. Rev. E 53, 1138 (1996).

[24] A.G. Kalocsai and J.W. Haus, Opt. Commun. 97, 239 (1993); Q. Guo, Quantum Opt. 5, 133 (1993).

[25] A.V. Buryak and Y.S. Kivshar, Phys. Lett. A 197, 407 (1995).

[26] C. Etrich, U. Peschel, F. Lederer, and B. Malomed, Phys. Rev. A 52, R3444 (1995); D. M. Baboiu, G. I. Stegeman, and L. Torner, Opt. Lett. 20, 2282 (1995); C. Balslev Clausen, P.L. Christiansen, and L. Torner, Opt. Commun. 136, 185 (1997).

[27] T. Gabler, Frauenhofer Institut für Angewandte Optik un Feinmechanik (private communication).

[28] M.F. Jørgensen, and P.L. Christiansen (unpublished).

[29] O. Bang, J. Opt. Soc. Am. B 14, 51 (1997).

[30] S. Trillo, S. Wabnitz, R. Chisari, and G. Cappellini, Opt. Lett. 17, 637 (1992).

[31] K.C. Rustagi, S.C. Mehendale, and S. Meenakshi, IEEE J. Quantum Electron. 18, 1029 (1982).

[32] A. Kobyakov and F. Lederer, Phys. Rev. A 54, 3455 (1996); A. Kobyakov, E. Schmidt, and F. Lederer, J. Opt. Soc. Am. B 14, 3242 (1997). 